\title{
668 LIPID-INSTRUCTED METABOLIC REWIRING UNLEASH THE ANTI-TUMOR POTENTIAL OF CD8+ T CELLS
}

${ }^{1}$ Teresa Manzo*, ${ }^{1}$ Carina Nava Lauveson, ${ }^{1}$ Teresa Maria Frasconi, ${ }^{1}$ Silvia Tiberti, ${ }^{2}$ Ignazio Caruana, ${ }^{1}$ Luigi Nezi, ${ }^{3}$ Philip Greenberg. 'European Institute of Oncology- IEO, Milan, Italy; ${ }^{2}$ University Children Hospital of Würzburg, Würzburg, Germany; ${ }^{3}$ Fred Hutchinson Cancer Research Center, Seattle, WA, United States

Background Adoptive cell therapy (ACT) harnesses the immune system to recognise tumor cells and carry out an anti-tumor function. However, metabolic constraints imposed by the tumour microenvironment (TME) suppress anti-tumor responses of CTL by reshaping their metabolism and epigenetic landscape. We have recently demonstrated that progressive accumulation of specific long-chain fatty acids (LCFAs) impair mitochondrial function and drives CD8 $+\mathrm{T}$ cell dysfunction. In this scenario, maintaining $\mathrm{T}$ cells in a less-differentiated state and with high metabolic plasticity during ex vivo $\mathrm{T}$ cell production and after infusion may have a strong therapeutic impact. Here, we propose a novel strategy to boost ACT efficacy by implementing $\mathrm{T}$ cell long-term functionality, metabolic fitness and preventing exhaustion through lipid-induced mitochondrial rewiring.

Methods We screen different LCFAs and assess their ability to shape CD8 $+\mathrm{T}$ cell differentiation using multi-parametric flow cytometry, proliferation and cytotoxic assays, together with a complete transcriptomic and epigenomic profiling. Metabolic reprogramming of lipid-treated CD $8+\mathrm{T}$ cell was examined by bioenergetic flux measurements paired with metabolomic and lipidomic analysis. Finally, the anti-tumor responses of lipidinstructed CD8 $\mathrm{T}$ cells was evaluated in a melanoma mouse model, known to poorly respond to immunotherapy.

Results LCFAs-treated CD8 + T cells are endowed with highly effector and cytotoxic features but still retaining a memorylike phenotype with decreased PD1 protein levels. Consistently, analysis of the bioenergetic profile and mitochondrial activity has shown that LCFA-instructed CD8 $+\mathrm{T}$ cells display a greater mitochondrial fitness. Thus, in vitro LCFA-instructed $\mathrm{CD} 8+\mathrm{T}$ cells are characterized by higher mitochondrial fitness, potent functionality, memory-like phenotype and PD-1 down-regulation, overall evoking the ideal $\mathrm{T}$ cell population associated with a productive anti-tumor response. The therapeutic potential of CD8 T cells lipid-induced metabolic rewiring was further confirmed in vivo. ACT performed with LCFA-reprogrammed CD8 T cells induces higher frequency of memory $\mathrm{T}$ cells, which show high polyfunctionality and mitochondrial function, decreased PD1 expression, ultimately resulting in improved tumor control. In addition, LCFAinduced metabolic rewiring during manufacturing of human CAR-redirected T cells, generated a CD8 + T cell memory-like population with higher mitochondrial fitness coupled with a much potent cytotoxic activity.

Conclusions These results suggest that LCFAs dictate the fate of CD8 + T cell differentiation and could be considered as a molecular switch to fine-tune memory $\mathrm{T}$ cell formation and metabolic fitness maintenance, linking lipid metabolism to anti-tumor surveillance. This will be of fundamental importance for a new generation of adoptive $\mathrm{T}$ cell-based therapies. Ethics Approval The experiments described were performed in accordance with the European Union Guideline on Animal Experiments and mouse protocols were approved by Italian Ministry of Health and the IEO Committee.

http://dx.doi.org/10.1136/jitc-2021-SITC2021.668 